\title{
Spontaneous parity violation and minimal Higgs models.
}

\author{
H. Chavez and J. A. Martins Simões, \\ Instituto de Física, \\ Universidade Federal do Rio de Janeiro, RJ, Brazil
}

\begin{abstract}
In this paper we present a model for the spontaneous breaking of parity with two Higgs doublets and two neutral Higgs singlets which are even and odd under $\mathcal{D}$-parity. The condition $v_{R} \gg v_{L}$ can be satisfied without introducing bidoublets and it is induced by the breaking of $\mathcal{D}$ parity through the vacuum expectation value of the odd Higgs singlet. Examples of left-right symmetric and mirror fermions models in grand unified theories are presented.

PACS: $12.60 . \mathrm{Cn}, 14.80 . \mathrm{Cp}, 12.10 . \mathrm{Dm}$
\end{abstract}

\section{Introduction}

Left-right symmetric models with spontaneous parity breaking offer a natural explanation for the parity asymmetry observed in nature. The gauge group $S U(2)_{L} \otimes S U(2)_{R} \otimes U(1)_{B-L}$ fixes the interactions and generalizes the standard electroweak theory. However the fundamental fermionic representation and the Higgs sector are not completely determined. For the fermions one can have two possibilities: new right-handed doublets, as in the earlier models [1, or new mirror fermions 2, 3. The Higgs sector has more possibilities and introduces more unknown parameters in the model. It is highly desirable to have the minimum number of unknown parameters in order to compare models and experimental data. A recent work in this direction was done by Brahmachari, Ma and Sarkar 4. With two Higgs doublets, $\chi_{R}$ and $\chi_{L}$, and a dimension five operator they have proposed a left-right model including fermion masses. Another mirror model with two Higgs doublets and two Higgs singlets was developed in ref.3. In both cases the condition

$$
v_{R} \gg v_{L}
$$

must be satisfied. The present experimental bound is $v_{R}>30 v_{L}$ [3]. Later on, Siringo 5 revived an earlier remark by Senjanovic and Mohapatra [6] that the above condition can not be satisfied in models with only two Higgs doublets. These remarks seems to leave open the possibility that only scalar bidoublets could break parity in a consistent way. This possibility exists but has the unpleasant feature of a large number of Higgs fields and undetermined parameters.

However, there is other elegant way 7 to produce the condition (1) by introducing one singlet Higgs which is odd under $\mathcal{D}$-parity. The difference with the $\mathcal{P}$-parity breaking is that in $\mathcal{D}$-parity the vacuum expectation value (VEV) 
of a parity odd field can be spontaneously broken without breaking the leftright symmetry. In consequence, the gauge coupling constants of $S U(2)_{R}$ and $S U(2)_{L}$ also can be different and left-handed and right-handed scalars can have different masses and VEVs.

In the present paper, we extend the previous analysis to include also a singlet Higgs field which is even under $\mathcal{D}$-parity and mixes with the odd field. We show that this mixing term also contribute to the hierarchy relation (1). We include in our study two examples of grand unified theories where Higgs singlets transforming under $\mathcal{D}$-parity are assigned to the L-R symmetric model and to a mirror fermion model.

\section{The scalar potential and the breaking of the L-R symmetry}

There are two forms of breaking parity spontaneously: the first is to identify the discrete symmetry $Z_{2}$ that interchanges the groups $S U(2)_{L}$ and $S U(2)_{R}$ of the Lorentz group $O(3,1)$ as the parity operator $\mathcal{P}$ that transforms the Higgs bosons $\chi_{L} \stackrel{\mathcal{P}}{\stackrel{P}{ }} \chi_{R}$ and also $W_{L} \stackrel{\mathcal{P}}{\longrightarrow} W_{R}$. So, when $S U(2)_{R}$ is broken in the symmetric L-R model, parity $\overleftrightarrow{\mathcal{P}}$ is also broken. The second possibility of spontaneously breaking the parity symmetry is through the VEV of an odd scalar field which preserves L-R symmetry. This type of parity is called $\mathcal{D}$-parity which is a generator of larger groups that contain the product $S U(2)_{L} \otimes S U(2)_{R}$ as a subgroup. This second possibility is very interesting because it allows $\left\langle\chi_{L}\right\rangle \ll\left\langle\chi_{R}\right\rangle$ with different coupling constants for $S U(2)_{L}$ and $S U(2)_{R}$ and different masses for the Higgs fields.

Our model for the scalar potential includes two doublets and two singlets Higgs fields. These singlets and doublets transforms under $\mathcal{D}$-parity as $S_{M} \mathcal{D} S_{M}$ ; $S_{D} \stackrel{\mathcal{D}}{\leftrightarrows}-S_{D}$ and $\chi_{L} \stackrel{\mathcal{D}}{\longleftrightarrow} \chi_{R}$, if in the model there are no CP violating terms or no complex Yukawa couplings. We propose the following invariant potential under $G_{3221}=S U(3)_{C} \otimes S U(2)_{L} \otimes S U(2)_{R} \otimes U(1)_{B-L}$ for the Higgs fields

$$
\begin{gathered}
V\left(\chi_{L}, \chi_{R}, S_{D}, S_{M}\right)=\mu^{2}\left(\chi_{L}^{\dagger} \chi_{L}+\chi_{R}^{\dagger} \chi_{R}\right)-\lambda_{\chi}\left(\chi_{L}^{\dagger} \chi_{L}+\chi_{R}^{\dagger} \chi_{R}\right)^{2}-m_{D}^{2} S_{D}^{2}- \\
\eta_{D} S_{D}^{3}-\lambda_{D} S_{D}^{4}-m_{M}^{2} S_{M}^{2}-\eta_{M} S_{M}^{3}-\lambda_{M} S_{M}^{4}+M_{D} S_{D}\left(\chi_{R}^{\dagger} \chi_{R}-\chi_{L}^{\dagger} \chi_{L}\right)+ \\
M_{M} S_{M}\left(\chi_{L}^{\dagger} \chi_{L}+\chi_{R}^{\dagger} \chi_{R}\right)+\lambda S_{D} S_{M}\left(\chi_{R}^{\dagger} \chi_{R}-\chi_{L}^{\dagger} \chi_{L}\right)+ \\
\left(\varepsilon_{D} S_{D}^{2}+\varepsilon_{M} S_{M}^{2}\right)\left(\chi_{L}^{\dagger} \chi_{L}+\chi_{R}^{\dagger} \chi_{R}\right)-\varkappa\left(\left(\chi_{L}^{4}\right)^{\dagger}+\chi_{L}^{4}+\left(\chi_{R}^{4}\right)^{\dagger}+\chi_{R}^{4}\right)
\end{gathered}
$$

Our motivation for this potential is the fact that $S_{M}$ and $S_{D}$ do not necessarily belong to the same irreducible multiplet of Higgs fields. In consequence it is also possible that these fields are mixed. If this is the case, when $\left\langle S_{D}\right\rangle=s_{D}$ and $\left\langle S_{M}\right\rangle=s_{M}$ the potential terms that contributes to the masses of Higgs fields $\chi_{L}$ and $\chi_{R}$ are

$$
\begin{gathered}
V_{\text {mass }}\left(\chi_{L}, \chi_{R}\right)=\left(\mu^{2}+\varepsilon_{D} s_{D}^{2}+\varepsilon_{M} s_{M}^{2}+M_{M} s_{M}\right)\left(\left|\chi_{L}\right|^{2}+\left|\chi_{R}\right|^{2}\right)+ \\
\left(M_{D} s_{D}+\lambda s_{D} s_{M}\right)\left(\left|\chi_{R}\right|^{2}-\left|\chi_{L}\right|^{2}\right)
\end{gathered}
$$


from which we obtain the masses

$$
\begin{aligned}
& m_{R}^{2}=\mu^{2}+\varepsilon_{D} s_{D}^{2}+\varepsilon_{M} s_{M}^{2}+M_{M} s_{M}+M_{D} s_{D}+\lambda s_{D} s_{M}, \\
& m_{L}^{2}=\mu^{2}+\varepsilon_{D} s_{D}^{2}+\varepsilon_{M} s_{M}^{2}+M_{M} s_{M}-M_{D} s_{D}-\lambda s_{D} s_{M} .
\end{aligned}
$$

Now we impose the hierarchy condition in the previous equations such that $m_{R}^{2}$ $\ll s_{D}^{2} \ll s_{M}^{2}$.It is necessary to indicate that $v_{L}$ breaks the electroweak symmetry and $v_{R}$ breaks the L-R symmetry close to the TeV scale. So we can have, for example $\left\langle\chi_{L}\right\rangle=v_{L} \sim m_{L} \sim 100 \mathrm{GeV}$ and, ; $\left\langle\chi_{R}\right\rangle=v_{R} \sim m_{R} \sim 10 \mathrm{TeV} \gg v_{L}$. It also must be noted that if $S_{D}$ and $S_{M}$ are in the same multiplet, then several possible mixing terms in the potential possibility are absent.

Let us now suppose that there are no CP violating terms and that all VEVs are considered to be real. With $\left\langle\chi_{L}\right\rangle=\left(\begin{array}{c}0 \\ v_{L}\end{array}\right),\left\langle\chi_{R}\right\rangle=\left(\begin{array}{c}0 \\ v_{R}\end{array}\right)$, it is possible to show that the minimum conditions for the potential are given by

$$
\begin{gathered}
\frac{\partial V}{\partial v_{L}}=2 v_{L}\left[\mu^{2}-2 \lambda_{\chi}\left(v_{L}^{2}+v_{R}^{2}\right)-M_{D} s_{D}+M_{M} s_{M}-\lambda s_{D} s_{M}+\right. \\
\left.\varepsilon_{D} s_{D}^{2}+\varepsilon_{M} s_{M}^{2}-4 \varkappa v_{L}^{2}\right]=0, \\
\frac{\partial V}{\partial v_{R}}=2 v_{R}\left[\mu^{2}-2 \lambda_{\chi}\left(v_{L}^{2}+v_{R}^{2}\right)+M_{D} s_{D}+M_{M} s_{M}+\lambda s_{D} s_{M}+\right. \\
\left.\varepsilon_{D} s_{D}^{2}+\varepsilon_{M} s_{M}^{2}-4 \varkappa v_{R}^{2}\right]=0,
\end{gathered}
$$

From these equations we have

$$
v_{L} \frac{\partial V}{\partial v_{R}}-v_{R} \frac{\partial V}{\partial v_{L}}=4 v_{L} v_{R}\left[M_{D} s_{D}+\lambda s_{D} s_{M}-2 \varkappa\left(v_{R}^{2}-v_{L}^{2}\right)\right]=0
$$

Now we require non trivial solutions such that $v_{L} \neq v_{R} \neq 0$. Thus we obtain the desired hierarchy

$$
v_{R}^{2}-v_{L}^{2}=\frac{s_{D}\left(M_{D}+\lambda s_{M}\right)}{2 \varkappa}
$$

A important point to be noted in the previous equation is that the breaking effect due to the singlet $S_{M}$ is sub-dominant with relation to $S_{D}$ that breaks $D$-parity when $\left\langle S_{D}\right\rangle=s_{D}$. Additionally, if $s_{D}=0$ then $D$-parity is conserved and the L-R symmetry condition is recovered, $v_{R}=v_{L}$. We have showed that the in our potential it is possible to construct models with L-R symmetry and producing an hierarchy between the breaking scale of $S U(2)_{R}$ and the electroweak scale. The main ingredient is the presence of two Higgs singlets to generate the minimum of the potential. The crucial point in this sense is the inclusion of the mixing term $\lambda S_{D} S_{M}\left(\chi_{R}^{\dagger} \chi_{R}-\chi_{L}^{\dagger} \chi_{L}\right)$ which is possible if $S_{M}$ and $S_{D}$ belong to different irreducible representations. As in the previous term, also the term $M_{D} S_{D}\left(\chi_{R}^{\dagger} \chi_{R}-\chi_{L}^{\dagger} \chi_{L}\right)$ breaks the L-R symmetry . It is also fundamental the fine tuning of the parameters of the model at the tree level in order to assure that $v_{R}$ do not destabilizes the $v_{L}$ value. Thus, from equations (5) - (7) we have

$$
m_{L}^{2}-2\left(\lambda_{\chi}+2 \varkappa\right) v_{L}^{2}=2 \lambda_{\chi} v_{R}^{2}
$$




\section{An $S O(10)$ L-R symmetric model.}

There is a known GUT context to embed the L-R symmetric model which is based on $S O(10)$ trough its maximal sub-group as done by the Pati-Salam [1] approach $G_{P S}=S U(4)_{C} \otimes S U(2)_{L} \otimes S U(2)_{R}$. The idea consists to break $\mathcal{D}$ parity below the breaking of $S O(10)$ as showed in the following breaking chain

$$
\begin{aligned}
S O(10) & \stackrel{S_{M}}{\longrightarrow} G_{P S} \otimes \mathcal{D} \quad \underset{S_{D}}{\longrightarrow} S U(3)_{C} \otimes S U(2)_{L} \otimes S U(2)_{R} \otimes U(1)_{B-L} \\
& \stackrel{\chi_{R}}{\longrightarrow} S U(3)_{C} \otimes S U(2)_{L} \otimes U(1)_{Y} \quad \stackrel{\chi_{L}}{\longrightarrow} S U(3)_{C} \otimes U(1)_{e . m},
\end{aligned}
$$

The quantum numbers for the Higgs representations that produces the pattern (11) are given in the Table 1.

Table 1

\begin{tabular}{|c|c|c|c|}
\hline$S_{M} \sim$ & $\{\mathbf{5 4}\}$ & $\supset[\mathbf{1}, \mathbf{1}, \mathbf{1}]$ & $\sim(\mathbf{1}, \mathbf{1}, \mathbf{1}, 0)$, \\
\hline$S_{D} \sim$ & $\{\mathbf{4 5}\}$ & $\supset[\mathbf{1 5}, \mathbf{1}, \mathbf{1}]$ & $\supset(\mathbf{1}, \mathbf{1}, \mathbf{1}, 0)$, \\
\hline$\chi_{R} \sim$ & $\left\{\mathbf{1 4 4}^{*}\right\}$ & $\supset[\mathbf{4}, \mathbf{1}, \mathbf{2}]$ & $\supset(\mathbf{1}, \mathbf{1}, \mathbf{2},-1)$, \\
\hline$\chi_{L} \sim$ & $\{\mathbf{1 4 4}\}$ & $\supset[\mathbf{4}, \mathbf{2}, \mathbf{1}]$ & $\supset(\mathbf{1}, \mathbf{2}, \mathbf{1},-1)$, \\
\hline
\end{tabular}

Higgs representations for the breaking chain (11).

Our notation is as follows: the representations between \{\} corresponds to $S O(10)$, [ ] corresponds to $G_{P S}$ and those with ( ) corresponds to $G_{3221}=$ $S U(3)_{C} \otimes S U(2)_{L} \otimes S U(2)_{R} \otimes U(1)_{B-L}$. In our model a different point from the approach of Ref. 8 is that we are using the singlet component $[\mathbf{1}, \mathbf{1}, \mathbf{1}]$ of $\{\mathbf{5 4}\}$ in order to break $S O(10)$ down to $G_{P S} \otimes \mathcal{D}$ because this is a symmetric representation $\mathcal{D}$-even 7 , 9], different of the $[\mathbf{1}, \mathbf{1}, \mathbf{1}]$ component of $\{\mathbf{2 1 0}\}$ which is $\mathcal{D}$-odd, as required of our analysis of the previous section. Note also that the neutral component $(\mathbf{1}, \mathbf{1}, \mathbf{1}, 0) \subset[\mathbf{1 5}, \mathbf{1}, \mathbf{1}] \subset\{\mathbf{4 5}\}$ of $S O(10)$ is $\mathcal{D}$-odd under $G_{3221}$. Thus, we are expecting that the VEV of $S_{D} \sim\{\mathbf{4 5}\}$, which will induce the breaking of the L-R symmetry, depend of the VEV of $S_{M}$. This choice could allow the breaking of the L-R symmetry close to the electroweak scale, let us say in the few $\mathrm{TeV}$ scale.

The $G_{3221}$ invariant Higgs potential of Equation.(2) could come from the following $S O(10)$ potential

$$
\begin{gathered}
\mathcal{L}=\mu^{2}\left(\mathbf{1 4 4}^{*} \times \mathbf{1 4 4}\right)+\lambda_{\chi}\left(\mathbf{1 4 4}^{*} \times \mathbf{1 4 4}\right)^{2}+m_{D}^{2}(\mathbf{4 5})^{2}+ \\
\eta_{D}(\mathbf{4 5})^{3}+\lambda_{D}(\mathbf{4 5})^{4}+m_{M}^{2}(\mathbf{5 4})^{2}+\eta_{M}(\mathbf{5 4})^{3}+\lambda_{M}(\mathbf{5 4})^{4} \\
+M_{D}(\mathbf{4 5})\left(\mathbf{1 4 4}^{*} \times \mathbf{1 4 4}\right)+M_{M}(\mathbf{5 4})\left(\mathbf{1 4 4}^{*} \times \mathbf{1 4 4}\right) \\
\quad+\lambda(\mathbf{5 4} \times \mathbf{4 5})\left(\mathbf{1 4 4}^{*} \times \mathbf{1 4 4}\right)+\left(\varepsilon_{D}(\mathbf{4 5})^{2}\right. \\
\left.+\varepsilon_{M}(\mathbf{5 4})^{2}\right)\left(\mathbf{1 4 4}^{*} \times \mathbf{1 4 4}\right)+\varkappa\left[\left(\mathbf{1 4 4}^{*}\right)^{4}+(\mathbf{1 4 4})^{4}\right] .
\end{gathered}
$$

Let us notice that the term $(\mathbf{5 4} \times \mathbf{4 5})\left(\mathbf{1 4 4} 4^{*} \times \mathbf{1 4 4}\right)$ is possible if the interactions between $\left(144^{*} \times \mathbf{1 4 4}\right)$ and $(\mathbf{5 4} \times \mathbf{4 5})$ are mediated by the gauge boson in the $\{\mathbf{4 5}\}$ or $\{\mathbf{5 4}\}$ representations.

The corresponding hypercharges are given by

$$
\frac{Y}{2}=T_{3 R}+\frac{B-L}{2}
$$


The left-handed ordinary fermions are contained in

$$
\begin{gathered}
\{\mathbf{1 6}\}_{i L}=\overbrace{q_{L}=\left(\begin{array}{l}
u \\
d
\end{array}\right)_{L}(\mathbf{3}, \mathbf{2}, \mathbf{1}, 1 / 3) \oplus l_{L}=\left(\begin{array}{l}
\nu \\
e
\end{array}\right)_{L}(\mathbf{1}, \mathbf{2}, \mathbf{1},-1)}^{[\mathbf{4}, \mathbf{2}, \mathbf{1}]} \\
\overbrace{q^{C}{ }_{L}=\left(\begin{array}{l}
d^{C} \\
u^{C}
\end{array}\right)_{L}\left(\mathbf{3}^{*}, \mathbf{1}, \mathbf{2},-1, \mathbf{2}\right]}^{\left[\mathbf{4}^{*},-1\right) \oplus l_{L}^{C}=\left(\begin{array}{l}
e^{C} \\
\nu^{C}
\end{array}\right)_{L}(\mathbf{1}, \mathbf{1}, \mathbf{2}, 1)}
\end{gathered}
$$

The Majorana and Dirac masses in our model can arise from dimension-5 effective operators such as

$$
\begin{aligned}
\mathcal{O}_{1} & =\frac{1}{\Lambda_{Q}}\left(\overline{q_{L}} \chi_{L}\right)\left(\overline{q_{R}} \chi_{R}^{*}\right), & \mathcal{O}_{2} & =\frac{1}{\Lambda_{Q}}\left(\overline{q_{L}} \chi_{L}^{*}\right)\left(q_{R} \chi_{R}\right), \\
\mathcal{O}_{3} & =\frac{1}{\Lambda_{D}}\left(\overline{l_{L}} \chi_{L}\right)\left(l_{R} \chi_{R}^{*}\right), & \mathcal{O}_{4} & =\frac{1}{\Lambda_{D}}\left(\overline{l_{L}} \chi_{L}^{*}\right)\left(l_{R} \chi_{R}\right), \\
\mathcal{O}_{5} & =\frac{1}{\Lambda_{M}}\left(l_{L} \chi_{L}^{*}\right)\left(l_{L} \chi_{L}^{*}\right), & \mathcal{O}_{6} & =\frac{1}{\Lambda_{M}}\left(l_{R} \chi_{R}^{*}\right)\left(l_{R} \chi_{R}^{*}\right),
\end{aligned}
$$

where $\Lambda_{Q, D, M}$ are the masses in the GUT scale. The first two operators $\mathcal{O}_{1}$ and $\mathcal{O}_{2}$ give the quark masses from which it is assumed the existence of the matter fields $\mathcal{Q}_{L, R} \sim(\mathbf{3}, \mathbf{1}, \mathbf{1}, 4 / 3) \subset(\mathbf{1 5}, \mathbf{1}, \mathbf{1}) \subset\{\mathbf{4 5}\}$ or $\{\mathbf{1 2 0}\}$ in order to generate the operator $\mathcal{O}_{1}$ and $\mathcal{M}_{L, R} \sim(\mathbf{3}, \mathbf{1}, \mathbf{1},-2 / 3) \subset(\mathbf{6}, \mathbf{1}, \mathbf{1}) \subset\{\mathbf{1 0}\}$, $\{\mathbf{1 2 6}\}$ or $\mathcal{M}_{L, R} \sim(\mathbf{3}, \mathbf{1}, \mathbf{1},-2 / 3) \subset(\mathbf{1 0}, \mathbf{1}, \mathbf{1}) \subset\{\mathbf{1 2 0}\}$ in order to generate the operator $\mathcal{O}_{2}$. On the other side, the operators $\mathcal{O}_{3}$ and $\mathcal{O}_{4}$ can be generated by the fermionic matter fields $\mathcal{P}_{L, R} \sim(\mathbf{1}, \mathbf{1}, \mathbf{1}, 0) \subset(\mathbf{1}, \mathbf{1}, \mathbf{1}) \subset\{\mathbf{5 4}\}$ and $\mathcal{S}_{L, R} \sim$ $(\mathbf{1}, \mathbf{1}, \mathbf{1},-2) \subset(\overline{\mathbf{1 0}}, \mathbf{1}, \mathbf{1}) \subset\{\mathbf{1 2 0}\}$. This is showed in Figure 1.
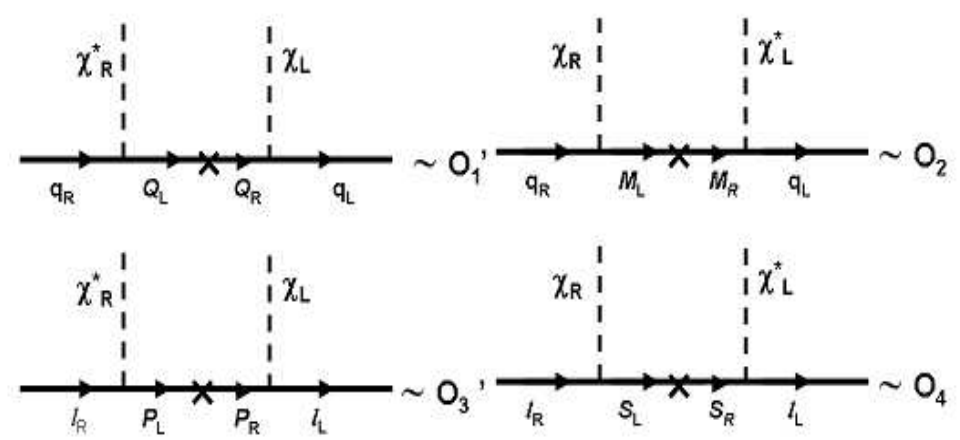

Figure 1: Diagrams producing the $\mathcal{O}_{1-4}$ operators of dimension-5.

To obtain the operators $\mathcal{O}_{5}$ and $\mathcal{O}_{6}$ let us observe that the operator $\left(\{\mathbf{1 6}\}_{L}\{\mathbf{1 6}\}_{L}\right)\left(\left\{\mathbf{1 4 4}^{*}\right\}\left\{\mathbf{1 4 4}^{*}\right\}\right)$ can be obtained through the mediation of the fermions in the $\{\mathbf{4 5}\}$ and $\{\mathbf{2 1 0}\}$ representations as it is showed in Figure 2. Operators of dimension- 5 of the Majorana type $\mathcal{O}_{5}$ can be obtained from diagram (2a), where it is necessary the inclusion of the fermionic matter term $\{\mathbf{4 5}\}$ in the 
$(\mathbf{1 5}, \mathbf{1}, \mathbf{1})$ component. This contribution to the neutrino mass is large because this fermion term corresponds to the GUT scale. To implement the see-saw mechanism we need to consider a term showed in Figure $2 \mathrm{~b}$ which include the D-parity effect through the Higgs singlet $\{\mathbf{4 5}\}$ and also a term that preserves it through the Higgs singlet $\{\mathbf{5 4}\}$. Details of the calculation and their relevance to generate magnetic moments for neutrinos and charged leptons will be presented elsewhere 10.

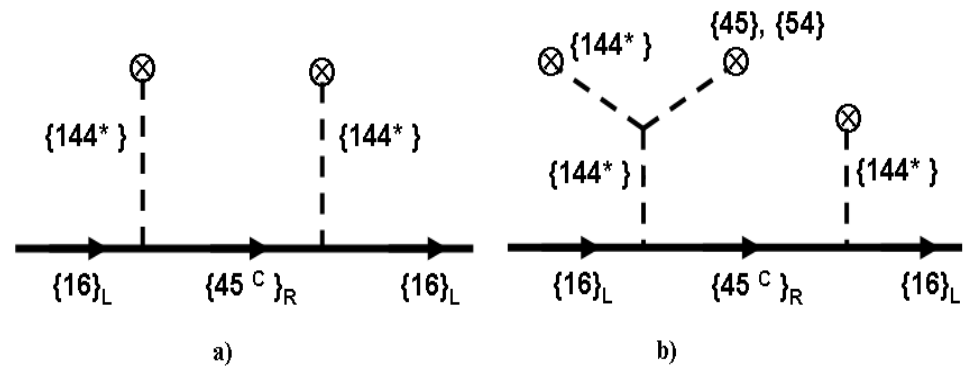

Figure 2: Diagrams for generating neutrino masses.

We have another possibility to embed $S U(2)_{L} \otimes S U(2)_{R} \otimes U(1)_{B-L}$ based in the breaking chain [1]

$$
\begin{gathered}
S O(10) \quad \stackrel{S_{M}}{\longrightarrow} G_{P S} \stackrel{S_{D}}{\longrightarrow} S U(3)_{C} \otimes S U(2)_{L} \otimes S U(2)_{R} \otimes U(1)_{B-L} \\
\stackrel{\chi_{R}}{\longrightarrow} S U(3)_{C} \otimes S U(2)_{L} \otimes U(1)_{Y} \quad \stackrel{\chi_{L}}{\longrightarrow} S U(3)_{C} \otimes U(1)_{e . m},
\end{gathered}
$$

The Higgs fields are given in table 2 . The component $[\mathbf{1}, \mathbf{1}, \mathbf{1}]$ of $\{\mathbf{2 1 0}\}$ is $\mathcal{D}$-odd.

Table 2

\begin{tabular}{|c|c|c|c|}
\hline$S_{M} \sim$ & $\{\mathbf{2 1 0}\}$ & $\supset[\mathbf{1}, \mathbf{1}, \mathbf{1}]$ & $\sim(\mathbf{1}, \mathbf{1}, \mathbf{1}, 0)$, \\
\hline$S_{D} \sim$ & $\{\mathbf{2 1 0}\}$ & $\supset[\mathbf{1 5}, \mathbf{1}, \mathbf{1}]$ & $\supset(\mathbf{1}, \mathbf{1}, \mathbf{1}, 0)$, \\
\hline$\chi_{R} \sim$ & $\left\{\mathbf{1 6}^{*}\right\}$ & $\supset[\mathbf{4}, \mathbf{1}, \mathbf{2}]$ & $\supset(\mathbf{1}, \mathbf{1}, \mathbf{2},-1)$, \\
\hline$\chi_{L} \sim$ & $\{\mathbf{1 6}\}$ & $\supset[\mathbf{4}, \mathbf{2}, \mathbf{1}]$ & $\supset(\mathbf{1}, \mathbf{2}, \mathbf{1},-1)$, \\
\hline
\end{tabular}

Higgs representations for the breaking chain (15).

If we use only the fields of $\{\mathbf{2 1 0}\}$ in order to produce the first two steps in (15), then the potential analogous to (12) has to be modified to

$$
\begin{gathered}
\mathcal{L}=\mu^{2}\left(\mathbf{1 6}^{*} \times \mathbf{1 6}\right)+\lambda_{\chi}\left(\mathbf{1 6}^{*} \times \mathbf{1 6}\right)^{2} \\
+m_{M}^{2}(\mathbf{2 1 0})^{2}+\eta_{M}(\mathbf{2 1 0})^{3}+\lambda_{M}(\mathbf{2 1 0})^{4}+M_{M}(\mathbf{2 1 0})\left(\mathbf{1 6}^{*} \times \mathbf{1 6}\right)+ \\
\varepsilon_{M}(\mathbf{2 1 0})^{2} \times\left(\mathbf{1 6}^{*} \times \mathbf{1 6}\right)+\varkappa\left[\left(\mathbf{1 6}^{*}\right)^{4}+(\mathbf{1 6})^{4}\right] .
\end{gathered}
$$

Then, following ref.[1], the masses of the Higgs doublets obtained from (16) are given by

$$
\begin{aligned}
& m_{R}^{2}=\mu^{2}+M_{M} s_{M}+\varepsilon_{M} s_{M}^{2}, \\
& m_{L}^{2}=\mu^{2}-M_{M} s_{M}+\varepsilon_{M} s_{M}^{2},
\end{aligned}
$$


As we have $\left\langle\chi_{L}\right\rangle=v_{L} \sim m_{L}$ and $\left\langle\chi_{R}\right\rangle=v_{R} \sim m_{R}$ we find, after the tunning of the model parameters, that $v_{L} \sim 100 \mathrm{GeV}$ and $v_{R} \sim G U T$. In fact,we have the relation

$$
v_{R}^{2}-v_{L}^{2}=\frac{M_{M} s_{M}}{2 \varkappa},
$$

From this equation we see that, due to the breaking of $\mathcal{D}$-parity in the GUT scale by the field $\mathcal{D}$-odd $[\mathbf{1}, \mathbf{1}, \mathbf{1}] \subset\{\mathbf{2 1 0}\}$, the breaking of the L-R symmetry is also induced close to the GUT scale. This is a different prediction of the model given by the breaking chain (11) in which it is possible that the breaking of L-R symmetry occurs close to the $\mathrm{TeV}$ scale. Other differences are also possible from the renormalization groups equations (RGE). This analysis for the breaking chain (15) was done in Ref.9 at one and two-loops on the gauge couplings. Some stages of the breaking chain (11) was analyzed in different papers 12 in the same context of the RGE. In fact, the use of the $G_{S M}$ singlets in the $\{\mathbf{1 6}\},\{\mathbf{1 2 6}\}$ and $\{\mathbf{1 4 4}\}$ representations would lead to the result that the unification of the $G_{S M}$ coupling constants is inconsistent with the low-energy data on these couplings. On the other side, the use of $\{\mathbf{5 4}\}$ in the breaking of $S O(10) \quad S_{M} \quad G_{P S} \otimes \mathcal{D}$ seems to be consistent with the experimental bound on the proton lifetime only at a marginal level 12 . We conclude that it would be necessary a more complete analysis of the RGE for the breaking chains along the lines of Ref. [13].

\section{An L-R model based in $\mathrm{SU}(7)$ with mirror fermions.}

In the L-R model with mirror fermions the particle content is described in Table 3 for the two first families with its its quantum numbers under $S U(3)_{C} \otimes$ $S U(2)_{L} \otimes S U(2)_{R} \otimes U(1)_{Y}$.

Table 3

\begin{tabular}{|c|c|}
\hline Ordinary fermions & Mirror fermions \\
\hline$l_{L}=\left(\begin{array}{c}\nu_{e} \\
e\end{array}\right)_{L},\left(\begin{array}{c}\nu_{\mu} \\
\mu\end{array}\right)_{L} \sim(\mathbf{1}, \mathbf{2}, \mathbf{1},-1)$ & $L_{R}=\left(\begin{array}{c}N_{E} \\
E\end{array}\right)_{R},\left(\begin{array}{c}N_{M} \\
M\end{array}\right)_{R} \sim(\mathbf{1}, \mathbf{1}, \mathbf{2},-1)$ \\
$e_{R}, \mu_{R} \sim(\mathbf{1}, \mathbf{1}, \mathbf{1},-2)$ & $E_{L}, M_{L} \sim(\mathbf{1}, \mathbf{1}, \mathbf{1},-2)$ \\
$\nu_{e R}, \nu_{\mu R} \sim(\mathbf{1}, \mathbf{1}, \mathbf{1}, 0)$ & $N_{E L}, N_{M L} \sim(\mathbf{1}, \mathbf{1}, \mathbf{1}, 0)$ \\
$\left(\begin{array}{c}u \\
d\end{array}\right)_{L},\left(\begin{array}{c}c \\
s\end{array}\right)_{L} \sim(\mathbf{3}, \mathbf{2}, \mathbf{1}, 1 / 3)$ & $\left(\begin{array}{c}U \\
D\end{array}\right)_{R},\left(\begin{array}{c}C \\
S\end{array}\right)_{R} \sim(\mathbf{3}, \mathbf{1}, \mathbf{2}, 1 / 3)$ \\
$u_{R}, c_{R} \sim(\mathbf{3}, \mathbf{1}, \mathbf{1}, 4 / 3)$ & $U_{L}, C_{L} \sim(\mathbf{3}, \mathbf{1}, \mathbf{1}, 4 / 3)$ \\
$d_{R}, s_{R} \sim(\mathbf{3}, \mathbf{1}, \mathbf{1},-2 / 3)$ & $D_{L}, S_{L} \sim(\mathbf{3}, \mathbf{1}, \mathbf{1},-2 / 3)$ \\
\hline
\end{tabular}

Some points should be observed. First, as $S U(3)_{C} \otimes S U(2)_{L} \otimes U(1)_{Y}$ is a maximal sub-group of $S U(5)$, then we have $S U(3)_{C} \otimes S U(2)_{L} \otimes S U(2)_{R} \otimes$ $U(1)_{Y} \subset S U(5) \otimes S U(2)_{R} \subset S U(7)$. In fact $14 \quad S U(5) \otimes S U(2) \otimes U(1)_{X}$ is a maximal sub-group of $S U(7)$ and we can assume $S U(2)$ to have the right chirality $S U(2)_{R}$.

A second point is that the mass terms of leptons $\overline{l_{e L}} \chi_{L} e_{R}$ require Higgs representations $\chi_{L} \sim(\mathbf{1}, \mathbf{2}, \mathbf{1}, 1)$. Similarly the mass terms of the mirror partners $\overline{L_{E R}} \chi_{R} E_{L}$, require $\chi_{R} \sim(\mathbf{1}, \mathbf{1}, \mathbf{2}, 1)$. Mixing terms of the type $\overline{e_{R}} S_{D} E_{L}$, 
$\overline{\nu_{R}} S_{D} N_{E L}$ need $S_{D} \sim(\mathbf{1}, \mathbf{1}, \mathbf{1}, 0)$. Mass terms of the Majorana type $\overline{l_{e L}} \widetilde{\chi_{L}} N_{E L}^{C}$ need $\widetilde{\chi_{L}} \sim(\mathbf{1}, \mathbf{2}, \mathbf{1},-1)$ and $\overline{L_{E R}} \widetilde{\chi_{R}} \nu_{e R}^{C}$ need $\widetilde{\chi_{R}} \sim(\mathbf{1}, \mathbf{1}, \mathbf{2},-1)$ in order to give mass to neutrinos. The $\overline{N_{E L}^{C}} S_{M} N_{E L}$ and $\overline{\nu_{e R}^{C}} S_{M} \nu_{e R}$ terms are possible with $S_{M} \sim(\mathbf{1}, \mathbf{1}, \mathbf{1}, 0)$. Now, let us search for the representations of $\chi_{L, R}, S_{D}$ and $S_{M}$ in the $S U(7)$ context [15]. The fermionic multiplet are in the anomaly free combination 1 [16] $\{\mathbf{1}\} \oplus\{\mathbf{7}\} \oplus\{\mathbf{2 1}\} \oplus\{\mathbf{3 5}\}$ corresponding to the spinor representation 64 of $S O(14)$ into which $S U(7)$ is embedded. In the previous multiplets, $\{\mathbf{2 1}\}$ is a 2 -fold, $\{\mathbf{3 5}\}$ is a 4 -fold and $\{\mathbf{7}\}$ is a 6 -fold of totally antisymmetric tensors.

Let us note that 64 can contain two families of ordinary fermions with its respective mirror partners, for example the electron and muon families as is showed in Table 3. The other families can be incorporated into other $\mathbf{6 4}$ spinorial representation. The branching rules for each component of the spinorial representation, under its sub-group $S U(5) \otimes S U(2)_{R}$, are [17]:

$$
\begin{gathered}
\{\mathbf{3 5}\}=\left[\mathbf{1 0}^{*}, \mathbf{1}\right] \oplus[\mathbf{1 0}, \mathbf{2}] \oplus[\mathbf{5}, \mathbf{1}], \\
\{\mathbf{2 1}\}=\left[\mathbf{1 0}^{*}, \mathbf{1}\right] \oplus\left[\mathbf{5}^{*}, \mathbf{2}\right] \oplus[\mathbf{1}, \mathbf{1}], \\
\{\mathbf{7}\}=[\mathbf{5}, \mathbf{1}] \oplus[\mathbf{1}, \mathbf{2}],
\end{gathered}
$$

and under $S U(3)_{C} \otimes S U(2)_{L} \otimes S U(2)_{R} \otimes U(1)_{Y}$ are

$$
\begin{aligned}
& \{\mathbf{3 5}\}=\underbrace{(\mathbf{1}, \mathbf{1}, \mathbf{1},-2)}_{e_{R}} \oplus \underbrace{(\mathbf{3}, \mathbf{1}, \mathbf{1}, 4 / 3)}_{u_{R}} \oplus \underbrace{(\mathbf{3}, \mathbf{2}, \mathbf{1}, 1 / 3)}_{\left(\begin{array}{c}
c \\
s
\end{array}\right)_{L}} \underbrace{(\mathbf{1}, \mathbf{1}, \mathbf{1},-2)}_{E_{L}} \oplus \\
& \underbrace{(\mathbf{1}, \mathbf{1}, \mathbf{1},-2)}_{M_{L}} \oplus \underbrace{(\mathbf{3}, \mathbf{1}, \mathbf{1}, 4 / 3)}_{U_{L}} \oplus \underbrace{(\mathbf{3}, \mathbf{1}, \mathbf{1}, 4 / 3)}_{C_{L}} \oplus \underbrace{\mathbf{3}, \mathbf{1}, \mathbf{1},-2 / 3)}_{s_{R}} \oplus \\
& \underbrace{(\mathbf{1 , 2 , 1 , - 1 )}}_{\left(\begin{array}{c}
\nu_{e} \\
e
\end{array}\right)_{L}} \underbrace{(3,1,2,1 / 3)}_{\left(\begin{array}{c}
U \\
D
\end{array}\right)_{R}} \underbrace{(3,1,2,1 / 3)}_{\left(\begin{array}{c}
C \\
S
\end{array}\right)_{R}}, \\
& \{\mathbf{2 1}\}=\underbrace{(\mathbf{1 , 1 , 1 , - 2 )}}_{\mu_{R}} \oplus \underbrace{(\mathbf{3}, \mathbf{1}, \mathbf{1}, 4 / 3)}_{c_{R}} \underbrace{(\mathbf{3 , 2} \mathbf{1}, 1 / 3)}_{\left(\begin{array}{c}
u \\
d
\end{array}\right)_{L}} \underbrace{(\mathbf{1 , 1 , 2 , - 1 )}}_{\left(\begin{array}{c}
N_{E} \\
E
\end{array}\right)_{R}} \oplus \\
& \underbrace{(\mathbf{1}, \mathbf{1}, \mathbf{2},-1)}_{\left(\begin{array}{c}
N_{M} \\
M
\end{array}\right)_{R}} \underbrace{(\mathbf{3}, \mathbf{1}, \mathbf{1},-2 / 3)}_{D_{L}} \oplus \underbrace{(\mathbf{3}, \mathbf{1}, \mathbf{1},-2 / 3)}_{S_{L}} \oplus \underbrace{(\mathbf{1}, \mathbf{1}, \mathbf{1}, 0)}_{N_{M L}} \\
& \{\boldsymbol{7}\}=\underbrace{(\mathbf{1}, \mathbf{2}, \mathbf{1},-1)}_{\left(\begin{array}{c}
\nu_{\mu} \\
\mu
\end{array}\right)_{L}} \underbrace{(\mathbf{3}, \mathbf{1}, \mathbf{1},-2 / 3)}_{d_{R}} \oplus \underbrace{(\mathbf{1}, \mathbf{1}, \mathbf{1}, 0)}_{N_{E L}} \oplus \underbrace{(\mathbf{1}, \mathbf{1}, \mathbf{1}, 0)}_{N_{M L}} . \\
& \{\mathbf{1}\}=\underbrace{(\mathbf{1}, \mathbf{1}, \mathbf{1}, 0)}_{\nu_{e R}} \text {. }
\end{aligned}
$$

${ }^{1}$ We are using $\{\quad\}$ for the $S U(7)$ components too. 
From the product $\{\mathbf{6 3}\} \otimes\{\mathbf{6 3}\}=\{\mathbf{1}\}+\{\mathbf{6 3}\} \oplus \ldots$, we obtain the Higgs representations producing the mass terms for the fermions in the spinorial multiplet $\{\mathbf{6 3}\}=\{\mathbf{7}\} \oplus\{\mathbf{2 1}\} \oplus\{\mathbf{3 5}\}$ of $S U(7)$. With the help of the branching rules (15) - (17), we take

$$
\begin{aligned}
\chi_{L} & \sim\left\{\mathbf{7}^{*}\right\} \supset(\mathbf{1}, \mathbf{2}, \mathbf{1}, 1), \quad \chi_{R} \sim\left\{\mathbf{2 1}^{*}\right\} \supset(\mathbf{1}, \mathbf{1}, \mathbf{2}, 1), \\
S_{D} & \sim\{\mathbf{2 1}\} \supset(\mathbf{1}, \mathbf{1}, \mathbf{1}, 0), \quad S_{M} \sim\{\mathbf{1}\} \sim(\mathbf{1}, \mathbf{1}, \mathbf{1}, 0) .
\end{aligned}
$$

Finally we can have the following breaking chain with two singlets and two doublets of Higgs representations:

$$
\begin{gathered}
S U(7) \underset{M_{M}}{S} S U(5) \otimes S U(2)_{R} \otimes \mathcal{D} \underset{S_{D}}{S} G_{S M} \otimes S U(2)_{R} \\
\stackrel{\chi_{R}}{\longrightarrow} S U(3)_{C} \otimes S U(2)_{L} \otimes U(1)_{Y} \underset{\chi_{L}}{\longrightarrow} S U(3)_{C} \otimes U(1)_{e . m} .
\end{gathered}
$$

The component of $\phi^{\alpha \beta}=\{\mathbf{2 1}\}$ that breaks $\mathcal{D}$-parity is given by $S_{D}=\phi^{67}$ which is odd under $\mathcal{D}$-parity [18] and the $S_{M}$ field being an $S U(7)$ singlet it preserves $\mathcal{D}$-parity.

We can write a $\mathrm{n} S U(7)$ invariant Higgs potential similar to the one given in Equation.(12) with the obvious changes $144 \rightarrow\left\{7^{*}\right\}, 45 \rightarrow\{21\}$, and $\mathbf{5 4} \rightarrow\{1\}$.

\section{Conclusions.}

In conclusion we have shown that parity can be spontaneously broken by a simple Higgs sector with two doublets and two singlets. The grand unified sector that contains this possibility is more restricted than other scenarios. One of the new proposed singlets can have a breaking scale not very far from the Fermi scale. A similar conclusion was recently found in a different approach for two doublets models [19. In the case of the model with mirror fermions it is possible a significant contribution to the magnetic moment of electrons and muons [10] due to couplings with the mirror fermions of the type $f\left(\overline{l_{L}} \chi_{L} e_{R}+\right.$ $\left.\overline{l_{R}} \chi_{R} E_{L}\right)+f^{\prime} \overline{e_{R}} E_{L} S_{D}$. This terms can give an important contribution to the muon anomaly and will be connected to the breaking of the Weinberg symmetry 20.

Acknowledgments: We thanks CNPq and FAPERJ for financial support.

\section{References}

[1] J. C. Pati and A. Salam, Phys. Rev. D 10 (1974) 275 ; R. N. Mohapatra and J. C. Pati, Phys. Rev. D 11 (1975) 566.

[2] J. Maalampi and M. Roos, Phys. Rept. 186, 53 (1990).

[3] Y. A. Coutinho, J. A. Martins Simões and C. M. Porto, Eur. Phys. J. C 18, 779 (2001); F. M. L. Almeida, Y. A. Coutinho, J. A. Martins Simões, J. Ponciano, A. J. Ramalho, S. Wulck and M. A. B. Vale,Eur. Phys. J. C 38, 115 (2004);J. A. Martins Simões and J. Ponciano, Eur. Phys. J. C 32S1, 91 (2004).

[4] B. Brahmachari, E. Ma and U. Sarkar, Phys. Rev. Lett. 91, 011801 (2003). 
[5] F. Siringo, Phys. Rev. Lett. 92 (2004) 119101.

[6] G. Senjanovic and R. N. Mohapatra, Phys. Rev. D 12 (1975) 1502.

[7] D. Chang, R. N. Mohapatra and M. K. Parida, Phys. Rev. Lett. 52(1984)1072, D. Chang, R. N. Mohapatra, J. M. Gibson, R. E. Marshak and M. K. Parida, Phys. Rev. D 31(1985)1718

[8] B. R. Desai, G. Rajasekaran and U. Sarkar, Phys. Lett. B 626(2005)167 hep-ph/0411099,

[9] R. N. Mohapatra, Fortsch. Phys. 31(1983)185.

[10] H. Chávez and J. A. Martins Simões hep-ph/0610231

[11] Kaushik Bhattacharya, C. R. Das, Bipin R. Desai, G. Rajasekaran and Utpal Sarkar, hep-ph/0601170, Phys. Rev. D 74(2006)015003.

[12] "Simple GUTs" , Giovanni Amelino-Camelia, Published in Proceedings of 9th International Seminar on High-Energy Physics: Quark 96, Yaroslavl, Russia, 5-11 May 1996; F Acampora, G. Amelino-Camelia, F. Buccella, O. Pisanti, L. Rosa and T. Tuzi, Nuovo Cimento A 108(1995)375.

[13] F. Csikor and I. Montvay, Phys. Lett. B 324, 412 (1994)

[14] R. Slansky, Phys. Rep. 79 (1981) 1.

[15] N. S. Baaklini, Phys. Rev. D 21(1980)1932, A. Umemura and K. Yamamoto, Progress of Theor. Phys. 66(1981)1430.

[16] K. Yamamoto, Mirror Fermions in the SU(7) GUT and Their Effects on Flavour Changing Process, Kyoto University, unpublished, 1983.

[17] Kyuwan Hwang, Jihn E. Kim, Phys. Lett. B 540 (2002) 289, M. Claudson, A. Yildiz and P. H. Cox, Phys. Lett. B 97(1980)224, Jihn E. Kim, Phys. Rev. D 23 (1981)2706.

[18] Jihn E. Kim, Phys. Rev. D 26 (1982)2009.

[19] F. Siringo and L. Marotta, hep-ph/0605276.

[20] One work in this direction was done in the $E_{6}$ model: Helder Chávez, Cristine N. Ferreira and José A. Helayel-Neto, Phys. Rev. D 74(2006)033006-1. 